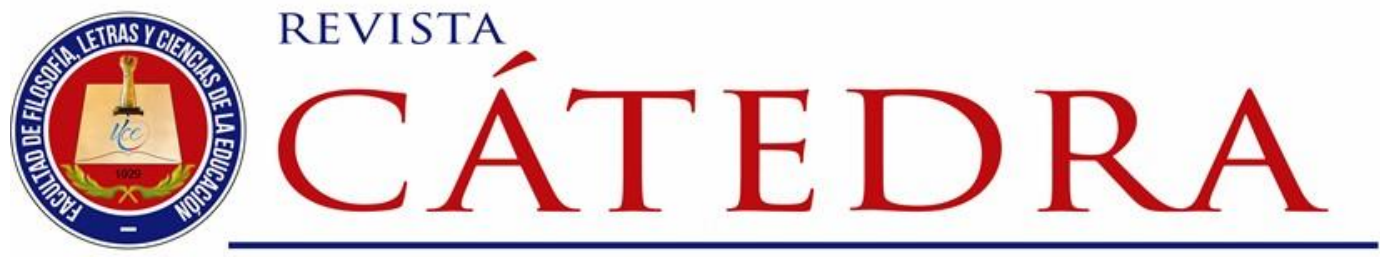

\title{
Análisis de la accesibilidad de los portales web de las instituciones educativas en la ciudad de Cuenca, Ecuador
}

\section{Accessibility analysis of the web portals of the educational institutions in Cuenca, Ecuador}

Milton Campoverde-Molina

Universidad Católica de Cuenca, Cuenca, Ecuador mcampoverde@ucacue.edu.ec https://orcid.org/0000-0001-5647-5150

Llorenç Valverde

Universitat de les Illes Balears, Mallorca, España

lvalverde@uib.cat

https://orcid.org/0000-0002-9163-568X

(Recibido: 13/03/2019; Aceptado: 21/03/2019; Versión final recibida: 16/04/2019)

Cita del artículo: Campoverde-Molina, M. y Valverde, L. (2019). Análisis de la accesibilidad de los portales web de las instituciones educativas en la ciudad de Cuenca, Ecuador. Revista Cátedra, 2(2), 55-75.

\section{Resumen}

Este artículo presenta un análisis de accesibilidad de los portales web de las instituciones educativas en la ciudad de Cuenca, Ecuador. El propósito de la investigación es evaluar la accesibilidad de los portales web con las Pautas de Accesibilidad para el Contenido Web (WCAG) 2.0 con un nivel de conformidad A y analizar su cumplimiento normativo. A partir de una revisión de diferentes fuentes bibliográficas se indagaron experiencias y resultados de investigaciones de accesibilidad web. Luego se presentan los resultados obtenidos de accesibilidad de 191 páginas web analizadas utilizando las herramientas en línea Examinator, TAW, Markup Validation Service y CSS Validation Service. Entre los resultados, se percibe que es necesario corregir errores en todos los portales web analizados de las instituciones educativas de la ciudad de Cuenca. Para establecer el nivel de cumplimiento se verifican los iconos que certifican la accesibilidad de los sitios por el World Wide Web Consortium (W3C) en HTML, CSS y sus niveles de conformidad A, AA y AAA. Con los datos obtenidos de la evaluación de las herramientas automáticas corroboramos el nivel de 
cumplimiento de los criterios de éxito de la WCAG 2.0 con un nivel de conformidad A. Se concluye que se requiere corregir errores en todos los portales web de las instituciones educativas de la ciudad de Cuenca analizadas. Por el promedio de errores encontrados, es más factible que las instituciones educativas rediseñen sus portales web, a fin de cumplir con las WCAG 2.0. También pueden mejorar el cumplimiento de las WCAG 2.0, con un arduo trabajo de revisión y corrección del código fuente de sus portales web.

\section{Palabras clave}

Accesibilidad web, discapacidades, educación, inclusión educativa, WCAG 2.0.

\section{Abstract}

This article shows the accessibility analysis of the websites of the educative institutions of Cuenca - Ecuador. The purpose of the research is to evaluate the accessibility of the websites with the Web Content Accessibility Guidelines (WCAG) 2.0 with a level of compliance A and analyze their regulatory compliance. Experiences and results of web accessibility research were investigated from a review of different bibliographic sources. The results obtained from the accessibility of 191 Web pages analyzed using the online tools Examiner, TAW, Markup Validation Service and CSS Validation Service are presented. Among the results, it is observed that it is necessary to correct errors in all the analyzed Web portals of the educational institutions of Cuenca. In order to establish the compliance level, the icons certifying the accessibility of the sites by the World Wide Web Consortium (W3C) in HTML, CSS and their conformity levels A, AA and AAA are verified. With the data obtained from the evaluation of the automatic tools, it is corroborated the compliance level of the success criteria of the WCAG 2.0 with a conformity level A. It is concluded that it is necessary to correct errors in all the web portals analyzed of the educational institutions of Cuenca. For the average number of errors found, it is more feasible for educational institutions to redesign their web portals in order to comply with the WCAG 2.0. They can also improve the fulfillment of the WCAG 2.0, with a hard work of revising and correcting the source code of their web portals.

\section{Keywords}

Web accessibility, disabilities, education, educational inclusion, WCAG 2.0.

\section{Introducción}

La accesibilidad web significa que las personas con algún tipo de discapacidad puedan hacer uso de la Web en las mismas condiciones que el resto de las personas. Al hablar de accesibilidad web se está haciendo referencia a un diseño que permita a personas con discapacidad percibir, entender e interactuar con la página o sitio que está navegando. La accesibilidad web también beneficia a otras personas, incluyendo personas de edad avanzada que han visto mermadas sus habilidades a consecuencia de los años. La Web es un recurso muy importante para diferentes aspectos de la vida: educación, empleo, gobierno, comercio, sanidad, entretenimiento y muchos otros. Es muy importante que la Web sea accesible para así proporcionar un acceso equitativo e igualdad de oportunidades a las personas con discapacidad. Una página web accesible puede ayudar a personas con discapacidad a que participen más activamente en la sociedad (World Wide Web Consortium, 2015). 
La ISO/IEC 40500: 2012 (International Organization for Standardization, 2012) pautas de accesibilidad del contenido web (WCAG) 2.0 cubre una amplia gama de recomendaciones para hacer que el contenido web sea más accesible. Seguir estas pautas hará que el contenido sea accesible para una gama más amplia de personas con discapacidades, que incluyen ceguera y visión deficiente, sordera y pérdida de audición, discapacidades de aprendizaje, limitaciones cognitivas, movimientos limitados, discapacidades del habla, sensibilidad fotográfica y combinaciones de estas. Seguir estas pautas también hará que el contenido web sea más usable para los usuarios en general. Los sitios, las tecnologías o las herramientas que no cumplan con estándares pueden crear barreras que excluyen a las personas del uso de la Web.

Ecuador, al igual que otros países (Instituto Ecuatoriano de Normalización, 2014), ha adoptado la norma ISO/IEC 40500:2012. Para dar seguimiento al cumplimiento obligatorio en el Ecuador de la norma NTE INEN-ISO/IEC 40500, se crea el reglamento técnico ecuatoriano RTE INEN 288 "Accesibilidad para el contenido web" (Instituto Ecuatoriano de Normalización, 2016) que entró en vigor el 8 de agosto de 2016. Este reglamento se aplica a los contenidos publicados en los sitios web del sector público y privado que presten servicios públicos. En su primera transitoria establece que, hasta el 8 de agosto de 2018, todos los sitios web ecuatorianos que presten un servicio público deben ser accesibles WCAG 2.0 nivel A. Conforme la WCAG 2.0 existen tres niveles de conformidad y 61 criterios de éxito que las páginas web deben cumplir A ( 25 criterios de éxito), AA (13 criterios de éxito) y AAA (23 criterios de éxito). De acuerdo al plazo establecido en la primera transitoria, los sitios web ecuatorianos deben haber cumplido con la norma de accesibilidad WCAG 2.0 nivel A en la actualidad. Para lograr un nivel de conformidad A (el mínimo), las páginas web deben haber cumplido los 25 criterios de éxito de la WCAG 2.0. Las instituciones públicas y privadas de la República del Ecuador junto con los desarrolladores web deben acoger e implementar el reglamento para permitir un acceso universal a la Web. Además, hasta el momento no se ha actualizado la normativa en Ecuador, pero es posible que lo haga en el futuro con las WCAG 2.1 (World Wide Web Consortium, 2018).

La educación está en un proceso evolutivo que va ajustándose a leyes, reglamentos y nuevas exigencias en la enseñanza-aprendizaje. Un aspecto clave es conseguir la inclusión y participación de todas las personas en el entorno educativo, tal como se requiere en el artículo 24 Educación de la Convención sobre los derechos de las personas con discapacidad (Naciones Unidas, 2006, pág. 19). Para lo cual, los portales web educativos también deben dar cumplimiento a lo establecido en el reglamento técnico ecuatoriano RTE INEN 288. Los administradores de los sitios web deben aplicar la normativa vigente de accesibilidad para que cualquier persona pueda hacer uso de sus portales web.

Según las estadísticas publicadas por el Consejo Nacional para la Igualdad de Discapacidades (CONADIS) con información del Ministerio de Salud Pública de las personas registradas con discapacidad en el Ecuador desde febrero 2016 hasta febrero 2019 (Consejo Nacional para la Igualdad de Discapacidades, 2019) se ha tenido un incremento de $11.01 \%$ en el Ecuador, mientras que en la provincia de Azuay desde octubre de 2016 hasta febrero de 2019 (Consejo Nacional para la Igualdad de Discapacidades, 2019) el incremento fue de $4.70 \%$ y, particularmente en la ciudad de Cuenca, el incremento corresponde al $5.69 \%$ (Consejo Nacional para la Igualdad de Discapacidades, 2019). Por otra parte, el $1 \%$ de las personas con discapacidades del Azuay se encuentran incluidas en la educación popular permanente, el $55 \%$ en educación regular y el $44 \%$ en educación especial (Consejo Nacional para la Igualdad de Discapacidades, 2015).

El propósito de esta investigación es evaluar la accesibilidad de los portales web de las instituciones educativas de la ciudad de Cuenca, Ecuador con las WCAG 2.0 y un nivel de

Licencia Creative Commons Atribución 4.0 Internacional (CC BY 4.0)

Revista Cátedra, 2 (2), pp. 55-75, mayo-agosto 2019. e-ISSN: 2631-2875

https://doi.org/10.29166/catedra.v2i2.1646 
conformidad A y analizar su cumplimiento normativo. Con este fin, buscamos respuestas a las siguientes preguntas:

- ¿Qué portales web de las instituciones educativas de la ciudad de Cuenca cumplen con las WCAG 2.0 y un nivel de conformidad A?

- ¿Cuál es el promedio de errores en los portales web de las instituciones educativas de la ciudad de Cuenca?

También, verificar si la entrada en vigor del reglamento técnico ecuatoriano y el cumplimiento del plazo de la primera transitoria han contribuido a mejorar la accesibilidad de los portales web educativos.

En cuanto a la estructura y contenido del artículo, en la sección 2 se presentan los conceptos relacionados con la investigación. En la sección 3, la revisión de diferentes fuentes bibliográficas de experiencias y resultados de investigaciones de accesibilidad web. En la sección 4, se detalla la metodología utilizada para desarrollar esta investigación. En la sección 5, se muestran los resultados de accesibilidad de los portales web de las instituciones educativas de la ciudad de Cuenca y su cumplimiento normativo. En la sección 6 , se establecen las conclusiones de acuerdo a los resultados obtenidos.

\section{Conceptos relacionados}

\subsection{Pautas de Accesibilidad para el Contenido Web (WCAG) 2.0}

Las WCAG 2.0 (World Wide Web Consortium, 2008) cubren un amplio rango de recomendaciones para crear contenido web más accesible. Se compone de 4 principios, 12 directrices y 61 criterios de cumplimiento (éxito), más un número no determinado de técnicas suficientes y técnicas de asesoramiento (Luján-Mora, 2018b):

1. Principio 1: Perceptible: la información y los componentes de la interfaz de usuario deben ser mostrados a los usuarios en formas que ellos puedan entender.

- Directriz 1.1: Texto alternativo: proporciona texto alternativo para el contenido que no sea textual, así podrá ser transformado en otros formatos que la gente necesite, como caracteres grandes, lenguaje braille, lenguaje oral, símbolos o lenguaje más simple.

- Directriz 1.2: Contenido multimedia dependiente del tiempo: proporcione alternativas sincronizadas para contenidos multimedia sincronizados dependientes del tiempo.

- Directriz 1.3: Adaptable: crear contenido que pueda ser presentado de diferentes formas sin perder ni información ni estructura.

- Directriz 1.4: Distinguible: facilitar a los usuarios ver y escuchar el contenido incluyendo la distinción entre lo más y menos importante.

2. Principio 2: Operable: los componentes de la interfaz de usuario y la navegación debe ser manejable.

- Directriz 2.1: Teclado accesible: poder controlar todas las funciones desde el teclado.

- Directriz 2.2 Tiempo suficiente: proporciona tiempo suficiente a los usuarios para leer y utilizar el contenido.

- Directriz 2.3: Ataques epilépticos: no diseñar contenido que pueda causar ataques epilépticos.

- Directriz 2.4: Navegación: proporciona formas para ayudar a los usuarios a navegar, a buscar contenido y a determinar dónde están estos. 
3. Principio 3: Comprensible: la información y las operaciones de usuarios deben ser comprensibles.

- Directriz 3.1: Legible: hacer contenido de texto legible y comprensible.

- Directriz 3.2 Previsible: hacer la apariencia y la forma de utilizar las páginas web previsibles.

- Directriz 3.3 Asistencia a la entrada de datos: los usuarios de ayuda evitarán y corregirán errores.

4. Principio 4: Robustez: el contenido deber ser suficientemente robusto para que pueda ser bien interpretado por una gran variedad de agentes de usuario, incluyendo tecnologías de asistencia.

- Directriz 4.1 Compatible: maximiza la compatibilidad con los agentes de usuario actuales y futuros, incluyendo tecnologías de asistencia.

\subsection{Niveles de conformidad}

Los niveles de conformidad son tres (World Wide Web Consortium, 2008):

- Nivel A: para la conformidad con el nivel A (el nivel mínimo de conformidad), la página web cumple con todos los criterios de éxito del nivel A, o se proporciona una versión alternativa conforme.

- Nivel AA: para la conformidad con el nivel AA, la página web satisface todos los criterios de éxito de nivel A y nivel AA, o se proporciona una versión alternativa que cumple con el nivel AA.

- Nivel AAA: para la conformidad con el nivel AAA, la página web satisface todos los criterios de éxito de nivel A, nivel AA y nivel AAA, o se proporciona una versión alternativa que cumple con el nivel AAA.

Cuando una página cumple con las Pautas WCAG 2.0 puede incluir en ella una declaración que indique a los usuarios que cumple con el W3C. El nivel de conformidad alcanzado se representa mediante un logotipo como se puede ver en el Cuadro 1.

\begin{tabular}{rrr}
\hline Nivel de Conformidad A & Nivel de Conformidad AA & Nivel de Conformidad \\
\hline W3C $\begin{array}{r}\text { WAI-A } \\
\text { WCAG 2.0 }\end{array}$ & WBC WAI-AA & AAA \\
\hline WCAG 2.0 & WBC WAI-AAA \\
WCAG 2.0
\end{tabular}

Si se utiliza algunos de los sellos anteriores, éste debe ir acompañado por la siguiente información (Confederación de Empresarios de Andalucía, 2010a):

- Fecha en que se revisó dicho cumplimiento.

- Título, versión y URI de las Pautas WCAG 2.0.

- Nivel de conformidad alcanzado (A, AA o AAA)

- Alcance: enumeración precisa de las páginas que cumplen con las Pautas WCAG 2.0.

- Listado de las tecnologías de las que depende el contenido.

En la Figura 1, se presentan los criterios de éxito, con sus niveles de conformidad por cada uno de los principios de la WCAG 2.0. 


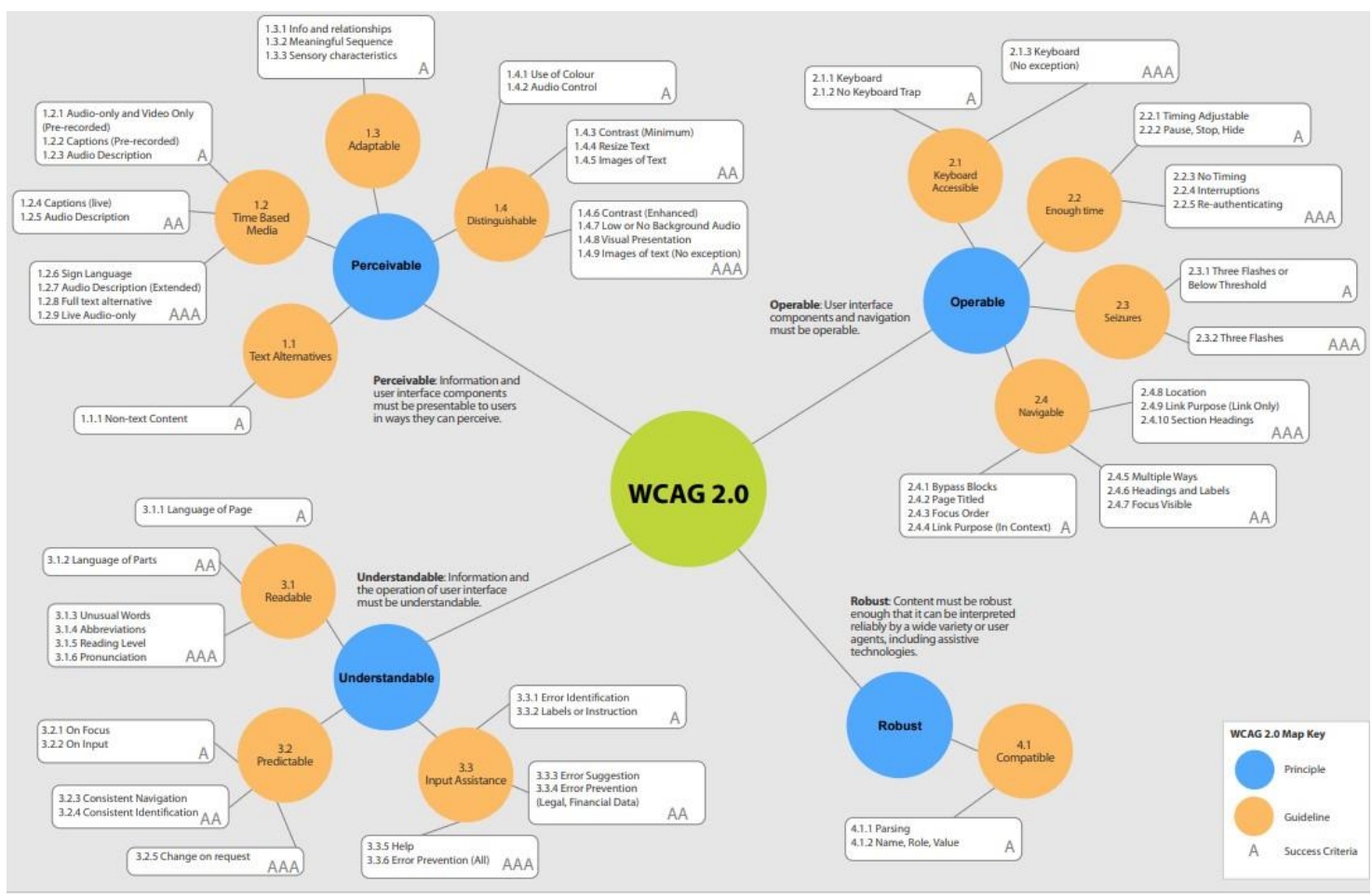

Figura 1. WCAG 2.0 Map (Stamford Interactive, 2012).

\subsection{Normativa de accesibilidad web en el Ecuador}

El 28 de enero de 2014 el Instituto Ecuatoriano de Normalización publicó en el registro Oficial No 171 la aprobación de la norma NTE INEN-ISO/IEC 40500 "Tecnología de la información - Directrices de accesibilidad para el contenido web del W3C (WCAG) 2.0 (ISO/IEC 40500:2012, IDT)" (Instituto Ecuatoriano de Normalización, Tecnología de la información - Directrices de accesibilidad para el contenido web del W3C (WCAG) 2.0, 2014). El 10 de febrero de 2016, el servicio Ecuatoriano de Normalización publicó el Reglamento Técnico Ecuatoriano RTE INEN 288 "Accesibilidad para el contenido web" (Instituto Ecuatoriano de Normalización, 2016). Este reglamento entró en vigor el 8 de agosto de 2016 y establece:

i. Aplica a los contenidos web publicados en los sitios web del sector público y privado que presten servicios públicos.

ii $\quad$ Se debe satisfacer por completo el nivel de conformidad AA, establecido en la norma NTE INEN-ISO/IEC 40500.

iii. Por último, se incluyen dos disposiciones transitorias sobre el plazo para cumplir con el reglamento y la norma:

- Transitoria Primera: los propietarios de los sitios web a los que se aplica este reglamento técnico tendrán un plazo de 2 años para adecuar sus sitios web existentes al momento de entrar en vigencia el reglamento de acuerdo al nivel de conformidad A de la norma NTE INEN vigente. El 8 de agosto de 2018, todos los sitios web ecuatorianos que presten un servicio público deben ser accesibles WCAG 2.0 nivel A.

- Transitoria Segunda: los propietarios de los sitios web a los que se aplica este reglamento técnico tendrán un plazo de 4 años para adecuar sus sitios web existentes al momento de entrar en vigencia el reglamento de acuerdo al nivel 
de conformidad AA. de la norma INEN vigente. El 8 de agosto de 2020, todos los sitios web ecuatorianos que presten un servicio público deben ser accesibles WCAG 2.0 nivel AA.

Además, el Reglamento Técnico Ecuatoriano RTE INEN 288 “Accesibilidad para el contenido web" emite un Régimen de Sanciones: "el propietario del sitio web que incumpla con lo establecido en este reglamento técnico recibirá las sanciones previstas en la Ley No. 200776 del Sistema Ecuatoriano de la Calidad, Ley Orgánica de Comunicación y demás leyes vigentes".

\subsection{Herramientas de evaluación de la accesibilidad web}

Las herramientas de evaluación de accesibilidad web son programas de software o servicios en línea que ayudan a determinar si el contenido web cumple con las pautas de accesibilidad (World Wide Web Consortium, Web Accessibility Evaluation Tools List, 2006). Una herramienta de este tipo nunca puede sustituir la revisión que realiza un experto en accesibilidad web, por lo que se deben emplear como un primer paso, pero no el único (Luján-Mora, 2018a). Aunque resultan de gran utilidad, se debe tener en cuenta que tienen ciertas limitaciones y no son capaces de identificar todos los problemas de accesibilidad. No son suficientes para asegurar que un sitio web es $100 \%$ accesible y por ello la revisión automática hay que completarla con otra manual (Confederación de Empresarios de Andalucía, 2010b).

\subsubsection{Examinator}

Examinator ${ }^{1}$ es un servicio en línea para evaluar de modo automático la accesibilidad de una página web, usando como referencia algunas técnicas recomendadas por las WCAG 2.0. Adjudica una puntuación entre 1 y 10 como un indicador rápido de la accesibilidad de las páginas y proporciona un informe detallado de las pruebas realizadas.

\subsubsection{Test de accesibilidad web (TAW)}

$\mathrm{TAW}^{2}$ es una herramienta automática en línea para analizar la accesibilidad de sitios web. El objetivo de TAW es comprobar el nivel de accesibilidad alcanzado en el diseño y desarrollo de páginas web con el fin de permitir el acceso a todas las personas independientemente de sus características diferenciadoras. Está destinada tanto a usuarios sin experiencia que quieren conocer el grado de accesibilidad de su sitio web como para administradores de sitios web, desarrolladores, diseñadores de páginas web, etc.

\subsubsection{Markup Validation Service}

El Markup Validation Service ${ }^{3}$ es un servicio gratuito del W3C que ayuda a verificar la validez de los documentos web escritos en lenguajes de marcado HTML o XHTML. Estos lenguajes están definidos por especificaciones técnicas, que generalmente incluyen una gramática (y vocabulario) formal legible por máquina. El acto de comparar un documento con estas restricciones se denomina validación, y esto es lo que hace el validador de marcado.

\footnotetext{
${ }^{1}$ Examinator: Evaluación de la accesibilidad web. Obtenido de http://examinator.ws/

2 TAW: Test de accesibilidad web. Obtenido de https://www.tawdis.net/

${ }^{3}$ Markup Validation Service: Servicio de Validación de Marcado W3C. Obtenido de https://validator.w3.org/about.html/
} 


\subsubsection{CSS Validation Service}

El CSS Validation Service ${ }^{4}$ del W3C es un software libre creado por el W3C para ayudar a los diseñadores y desarrolladores web a validar Hojas de Estilo en Cascada (CSS). Lo que hace esta herramienta es ayudar a las personas que escriben CSS a comprobar, y corregir si es necesario, sus Hojas de Estilo en Cascada. No solo compara las hojas de estilo con las especificaciones CSS, sino también ayuda a encontrar errores comunes, errores tipográficos, o usos incorrectos de CSS y algún riesgo en cuanto a usabilidad.

\section{Trabajos relacionados}

En el año 2016, una investigación realizó la evaluación de 15 portales web, clasificados en 7 hospitales, 5 clínicas y 3 centros médicos utilizando la herramienta en línea TAW. En los resultados se determina que las instituciones de salud analizadas no cumplen con las directrices de las WCAG 2.0 con un nivel de conformidad A. Por lo tanto, concluyen los autores que los portales web no son accesibles y su mayor problema está en el principio de robustez (Campoverde-Molina, Vizñay-Durán y Reyes-Espinosa, 2016).

Un estudio presentado en el año 2016 mostró los resultados preliminares de un análisis de accesibilidad de 31 portales web de las unidades educativas y colegios de la ciudad de Cuenca, Ecuador. En los resultados se estableció que los portales no cumplen con las directrices de las WCAG 2.0 con un nivel de conformidad A y su mayor problema está en el principio de robustez. Adicionalmente, el autor concluye que el $92 \%$ de los problemas en los portales web son advertencias, el $6 \%$ son errores y el $2 \%$ son puntos no verificados que requieren de un análisis manual completo (Campoverde-Molina, 2016).

En el año 2016, una investigación realizó la evaluación de accesibilidad de las páginas principales de 20 universidades de todo el mundo clasificadas por Webometrics. De los resultados, los autores concluyen que la mayoría de los sitios web probados no alcanzan un nivel aceptable de cumplimiento. Las universidades con alto prestigio académico no muestran un mayor nivel de accesibilidad web. Al realizar la evaluación de los sitios web de las universidades, los autores han identificado que existen grandes barreras para un gran número de usuarios (Acosta-Vargas, Luján-Mora y Salvador-Ullauri, 2016).

En el año 2016, una investigación realizó un estudio de 328 muestras de sitios web de alojamientos con familias en Malasia utilizando la herramienta de evaluación automatizada (Achecker) con las WCAG 2.0. Los resultados muestran que hay 6 errores críticos de accesibilidad principales:

- Contenido no textual: img, texto alternativo (1.1.1).

- Relaciones de información: entrada (1.3.1).

- Contraste: enlace, color de texto (1.4.3).

- Enlace propósito: ancla, texto (2.4.4).

- Idioma de la página: HTML (3.1.1).

- Etiquetas o instrucciones: cuerpo, entrada (3.3.2).

$1.1{ }^{4}$ CSS Validation Service: Acerca del Validador de CSS. Obtenido de https://jigsaw.w3.org/cssvalidator/about.html

Licencia Creative Commons Atribución 4.0 Internacional (CC BY 4.0)

Revista Cátedra, 2 (2), pp. 55-75, mayo-agosto 2019. e-ISSN: 2631-2875

https://doi.org/10.29166/catedra.v2i2.1646 
Además, esta investigación proporciona recomendaciones para mejorar los niveles de accesibilidad de los sitios web de los alojamientos con familias en Malasia (Rahim Wan Mohd Isa, Hakim Suhaimi, Ariffrn, Fatimah Ishak y Mohd Ralim, 2016).

En el año 2016, se realizó la evaluación de accesibilidad de 5 portales web del Estado peruano a partir de su legislación, en las recomendaciones WCAG 1.0 y WCAG 2.0 del W3C. Para tal fin, los autores identificaron los portales que tienen mayor acceso e importancia en el momento de la investigación. Los resultados arrojan un conjunto de deficiencias como: errores del color, audio, texto, contenido no textual y alt en imágenes (el atributo alt en HTML se utiliza para describir o contextualizar una imagen a personas con discapacidad visual que utilizan lectores de pantalla), errores en los componentes de interfaz presentados al usuario, enlaces sin contenidos y el orden de navegación mediante tabulación resulta inapropiado (Sam-Anlas y Stable-Rodríguez, 2016).

En un estudio en el año 2017, se realizó la evaluación de las políticas de accesibilidad web adoptadas por 51 sitios web de universidades del mundo, que están clasificadas por Webometrics. Los autores concluyen que 44 de las 51 universidades evaluadas no alcanzaron un nivel aceptable de políticas de accesibilidad web. Los resultados muestran que, aunque la mayoría de las universidades tienen políticas de accesibilidad en la web, la mayoría de las políticas tienen serias deficiencias. Las deficiencias identificadas en este trabajo son lo suficientemente graves como para violar los derechos legales de accesibilidad de las personas con discapacidades. Las cuales representan una amenaza para estas personas que pueden demandar contra los sitios web según las leyes y regulaciones ejecutadas en cada país (Acosta-Vargas, Luján-Mora y Salvador-Ullauri, 2017).

En otra investigación, se evaluó la accesibilidad de 44 universidades ecuatorianas usando el software en línea Examinator. De los resultados obtenidos los autores concluyeron que los sitios web de las instituciones de educación superior no son accesibles. Ninguna de las universidades evaluadas cumple con el Reglamento Técnico Ecuatoriano RTE INEN 288 el cual está vigente y es de cumplimiento obligatorio para las instituciones de educación superior. El puntaje promedio de accesibilidad de las páginas web analizadas es de 5.0 sobre 10. A partir de los 54 errores de accesibilidad que la herramienta Examinator identificó y los 8,970 elementos en los cuales se han incurrido en dichos errores, se concluye que: el $59 \%$ corresponden a fallos en el principio de accesibilidad perceptible, $24 \%$ al principio operable, el $10 \%$ al principio robusto y el $7 \%$ al principio comprensible; el $50 \%$ corresponden a errores en el nivel de conformidad A, y el $25 \%$ en cada uno de los niveles de conformidad AA y AAA respectivamente (Acosta y Luján-Mora, 2017).

En otra investigación, se manifestó que la accesibilidad de los sitios web es una forma sencilla de que todos puedan acceder a la información del sitio y pueda entenderse fácilmente. En los resultados del análisis de los 13 sitios web de universidades analizadas en Java Occidental, Indonesia, se presentó en un gráfico que muestra la tasa de errores de cada universidad. Los errores que ocurrieron en casi todos los sitios web fueron: contenido sin texto, información y relaciones, título de la página, propósito del enlace, idioma de la página, en la entrada, etiquetas e instrucciones, análisis y nombre, función, criterios de valor (Arasid et al., 2018).

En una investigación en el año 2018, se describieron los problemas de accesibilidad web identificados en 348 sitios web de universidades de América Latina según su clasificación en Webometrics. Los resultados muestran que los sitios web de las universidades tienen problemas frecuentes relacionados con la falta de texto alternativo en las imágenes. Se encontró que los sitios web de las universidades analizadas violan los requisitos de accesibilidad web basados en WCAG 2.0. Los numerosos problemas identificados en los

Licencia Creative Commons Atribución 4.0 Internacional (CC BY 4.0)

Revista Cátedra, 2 (2), pp. 55-75, mayo-agosto 2019. e-ISSN: 2631-2875

https://doi.org/10.29166/catedra.v2i2.1646 
sitios web indican que es necesario fortalecer las políticas de accesibilidad web en cada país y aplicar estándares para hacer que los sitios web sean más inclusivos (Acosta-Vargas, Acosta y Luján-Mora, 2018).

La investigación de Ortiz Ruiz (2019) tuvo como objetivo analizar políticas y normativas de Chile que orientan el diseño web para garantizar un nivel de accesibilidad acorde a la sociedad actual. En esta investigación se estipula que existen leyes y reglamentos de los derechos de las personas con discapacidad que tiene como exigencia un nivel AA para sitios gubernamentales elaborados en base a las WCAG 2.0 del W3C. A partir de estas referencias, se evaluaron las páginas iniciales desarrolladas por el Ministerio de Educación de acceso de docentes, estudiantes, apoderados, padres y público en general. Todos resultaron ser sitios no accesibles, siendo las debilidades más recurrentes la ausencia de textos alternativos en imágenes, videos u otros elementos que lo exigen y enlaces poco claros para una navegación asertiva, exigencias básicas de accesibilidad.

En una investigación del 2019, se dio a conocer que en la corrección de errores en el diseño de los sitios web se debe aplicar una metodología correcta para evitar productos de mala calidad. De esta manera, la evaluación es un proceso que, además de visibilizar los distintos errores, se convierte en una herramienta de control de calidad que permite desarrollar productos confiables y usables. No obstante, la evaluación comparativa nos permite determinar los errores con mayor presencia en cada uno de los sitios en estudio, permitiéndonos así identificar las áreas que necesitan mayor atención (Rodríguez V., Rosas Chávez y Suárez Naranjo, 2019).

\section{Metodología}

A continuación, se describen los pasos utilizados en esta investigación:

1. Selección de las instituciones educativas: se utilizó la información del período 2017-2018 del Archivo Maestro de Instituciones Educativas (AMIE) del Ministerio de Educación del Ecuador (Ministerio de Educación del Ecuador, 2018).

2. Selección de una muestra representativa: se seleccionaron 29 portales web con el dominio.edu.ec. Según los tipos de dominio en Ecuador (Network Information Center, 2019), el nombre de dominio.edu.ec es "solo para entidades, instituciones u organizaciones educativas, como colegios, universidades, escuelas e institutos registrados en Ecuador". De cada uno de los portales web se seleccionaron 7 páginas web de muestra y los que tienen menos de 7 todas sus páginas web.

3. Evaluación de las páginas seleccionadas de los portales web: para determinar los errores de accesibilidad de las 29 páginas principales y 162 páginas web se utilizaron las herramientas en línea Examinator, TAW, Markup Validation Service y CSS Validation Service. Las páginas principales son la primera impresión y la carta de presentación que se le da al usuario que visita un sitio web. Además, estas páginas permiten a los usuarios desplazarse entre los demás sitios de los portales web.

4. Nivel de cumplimiento: para establecer el nivel de cumplimiento se verificó los iconos que certifiquen la accesibilidad de los sitios por la W3C en HTML, CSS y sus niveles de conformidad A, AA y AAA. A partir de los datos obtenidos de la evaluación de las herramientas automáticas se corrobora el nivel de cumplimiento de los criterios de éxito de la WCAG 2.0 con un nivel de conformidad A. 


\section{Resultados}

\subsection{Selección de los portales web según el dominio.edu.ec}

El Ministerio de Educación del Ecuador (2018) en el AMIE, recoge datos de las instituciones públicas y privadas (estudiantes, docentes, infraestructura, entre otros) a nivel nacional y territorial al inicio y al final del año escolar. La información está disponible a partir del periodo 2009-2010 al 2017-2018. Con la información del período 2017-2018 se ha determinado que en la ciudad de Cuenca - Ecuador existen 529 establecimientos entre centros de educación, escuelas, instituciones educativas. Lo cual ha permitido seleccionar 29 portales web con el dominio.edu.ec como se puede ver en el Cuadro 2.

\begin{tabular}{|c|c|c|c|}
\hline № & Abreviaturas & Instituciones educativas & URL Portal web \\
\hline 1 & aaschool & $\begin{array}{l}\text { Escuela de Educación Básica Particular } \\
\text { Asian American School }\end{array}$ & www.aaschool.edu.ec \\
\hline 2 & adistanciabg & $\begin{array}{l}\text { Colegio de Bachillerato Particular Bill } \\
\text { Gates }\end{array}$ & colegioadistanciabg.edu.ec/index.php \\
\hline 3 & amauta & AMAUTA & www.amauta.edu.ec \\
\hline 4 & americancollege & Unidad Educativa American School & www.americanschool.edu.ec \\
\hline 5 & uepcc & $\begin{array}{l}\text { Unidad Educativa Particular Católica de } \\
\text { Cuenca }\end{array}$ & plantelesanexos.ucacue.edu.ec \\
\hline 6 & benignomalo & Unidad Educativa Benigno Malo & www.colegiobenignomalo.edu.ec \\
\hline 7 & Casc & Colegio Alemán Stiehle de Cuenca & www.casc.edu.ec \\
\hline 8 & catalinas & $\begin{array}{l}\text { Unidad Educativa Particular Rosa de } \\
\text { Jesús Cordero }\end{array}$ & www.catalinas.edu.ec \\
\hline 9 & comilcue & Unidad Educativa Abdón Calderón & www.comilcue.edu.ec \\
\hline 10 & continental & Unidad Educativa Continental & www.continental.edu.ec \\
\hline 11 & delasallecuenca & $\begin{array}{l}\text { Unidad Educativa Particular Hermano } \\
\text { Miguel de la Salle }\end{array}$ & www.delasallecuenca.edu.ec \\
\hline 12 & herlindatoral & Unidad Educativa Herlinda Toral & www.herlindatoral.edu.ec \\
\hline 13 & laasuncion & Unidad Educativa la Asunción & www.laasuncion.edu.ec \\
\hline 14 & latinoamericano & Unidad Educativa Latinoamericano & www.latinoamericano.edu.ec \\
\hline 15 & madrid & Unidad Educativa Madrid & www.unidadeducativamadrid.edu.ec \\
\hline 16 & manueljcalle & $\begin{array}{l}\text { Unidad Educativa del Milenio Manuel J } \\
\text { Calle }\end{array}$ & www.colegiomanueljcalle.edu.ec \\
\hline 17 & mariorizzini & Unidad Educativa Mario Rizzini & www.mariorizzini.edu.ec \\
\hline 18 & santana & Unidad Educativa Santana UNESA & www.santana.edu.ec \\
\hline 19 & Sscc & $\begin{array}{l}\text { Unidad Educativa Particular Sagrados } \\
\text { Corazones }\end{array}$ & www.sscc-cuenca.edu.ec \\
\hline 20 & sudamericano & Unidad Educativa Sudamericano & www.sudamericano.ec \\
\hline 21 & Uebi & $\begin{array}{ll}\text { Unidad Educativa } & \text { Bilingüe } \\
\text { Interamericano } & \\
\end{array}$ & www.uebi.edu.ec \\
\hline 22 & Uelac & $\begin{array}{l}\text { Unidad Educativa Liceo Americano } \\
\text { Católico }\end{array}$ & www.uelac.edu.ec \\
\hline 23 & Uemo & Unidad Educativa Misioneros Oblatos & www.uemo.edu.ec \\
\hline 24 & ueoblatas & $\begin{array}{l}\text { Unidad Educativa Particular Corazón de } \\
\text { María }\end{array}$ & www.ueoblatas.edu.ec \\
\hline 25 & ueporvenir & Unidad Educativa Porvenir & www.ueporvenir.edu.ec \\
\hline 26 & Uets & Unidad Educativa Técnico Salesiano & www.uets.edu.ec \\
\hline 27 & uesmacuenca & $\begin{array}{l}\text { Unidad Educativa Salesiana María } \\
\text { Auxiliadora }\end{array}$ & www.uesmacuenca.edu.ec \\
\hline 28 & unidadborja & Unidad Educativa Particular Borja & www.unidadborja.edu.ec \\
\hline 29 & verbo & Unidad Educativa Cristiana Verbo & www.verbo.edu.ec \\
\hline
\end{tabular}

Cuadro 2. Portales web de las instituciones educativas de la ciudad de Cuenca

\subsection{Selección de páginas web}

Luego de haber realizado el análisis de las páginas web que contienen los portales web de las instituciones educativas de la ciudad de Cuenca, determinamos que, las páginas web a 
las que los estudiantes acceden con más frecuencia para consultar o realizar una actividad académica es:

1. aaschool: página principal, el campus, tour virtual, la educación, idiomas, servicios y contáctanos.

2. adistanciabg: página principal, información académica (8vo básica), consejería estudiantil, secretaría, inglés, aula virtual colegio y bachillerato.

3. amauta: página principal, Ecuador, quienes somos, alojamientos con familias, programas, FAQS y contáctanos.

4. americancollege: página principal, proceso de admisión, becas, aranceles, inscripciones, aula virtual y biblioteca virtual.

5. benignomalo: página principal, estudiantes, contacto, formulario de registro, página de videos, página de eventos y biblioteca virtual.

6. casc: página principal, kindergarten, primaria, secundaria, actividades complementarias, admisiones y contáctenos.

7. catalinas: página principal, docentes, representantes y programa diploma.

8. comilcue: página principal, inicio de sesión de notas, inicio de sesión ingreso de tareas y contáctanos.

9. continental: página principal, información académica, aula virtual, distributivo, misión y visión, web mail y nuestros docentes.

10. delasallecuenca: página principal, quienes somos, oferta académica, estructura académica, estudiantes, deportes y contáctenos.

11. herlindatoral: página principal, ideario, secciones, misión, galería, noticias y contáctenos.

12. laasuncion: página principal, unidad educativa la asunción, ingreso sistema académico, noticias y eventos, revista indicios, aula virtual y contacto.

13. latinoamericano: página principal, preparatoria, básica elemental, básica superior, bachillerato, academia y básica media.

14. madrid: página principal, nuestra historia, misión y visión, ubicación, calendario y historia.

15. manueljcalle: página principal, inicio de sesión de notas y monografía.

16. mariorizzini: página principal, niveles educativos, horario de clases, descarga aplicaciones para móviles, registro, ubicación y contáctanos.

17. santana: página principal, porque santana, programas y proyectos, noticias y novedades, bachillerato internacional, ingreso sistema de calificaciones y escuela abierta.

18. sscc: página principal, oferta académica, noticias, galería, contacto, calendario y biblioteca.

19. sudamericano: página principal, oferta académica, noticias, admisiones, contacto, inicio de sesión de notas y tarifas educativas.

20. uebi: página principal, eventos, aula virtual, galería, contactos, personal administrativo y personal docente.

21. uelac: página principal, nosotros, galería de imágenes, contáctenos, noticias, horario inicial primero de básica y eventos.

22. uemo: página principal, nosotros, preparatoria, básica, superior, bachillerato y distributivo.

23. ueoblatas: página principal, información académica, contacto, club de periodismo, inicio de sesión notas, DECE y políticas institucionales.

24. uepcc: página principal, información, inscripciones primero de básica hasta bachillerato, inscripción inicial, matrículas y cronograma de matrículas. 
25. ueporvenir: página principal, misión y visión, horario de atención padres de familia, ubicación, autoridades, contacto y seguimiento académico.

26. uesmacuenca: página principal, educación virtual (página ingreso), información académica, noticias, comunicaciones, bachillerato en ciencias y perfil educación básica.

27. uets: página principal, docentes, datos informativos, ubicación, especializaciones, infraestructura e inicio de sesión aula virtual.

28. unidadborja: página principal, admisiones, nivel inicial, básica elemental y media, básica y superior, talleres y cursos y contáctenos.

29. verbo: página principal, quienes somos, instalaciones, noticias, contactos, libretas y ubicación.

Teniendo como resultado 191 páginas web de los portales de las instituciones educativas de la ciudad de Cuenca como objeto de estudio. Las páginas como notas, ingreso de tareas y aula virtual solo se realizará el análisis del inicio de sesión por no contar con las contraseñas para el ingreso y evaluación de sus contenidos.

\subsection{Evaluación páginas principales}

Con la finalidad de evaluar la accesibilidad de las páginas principales se utiliza la herramienta Examinator que presenta una batería completa de 100 pruebas automáticas, que resultan convenientes para una primera aproximación hacia la accesibilidad web. Se ha identificado que las páginas web de las instituciones educativas no fueron diseñadas con una visión de accesibilidad web, por lo que el uso de herramientas automáticas permite identificar los errores frecuentes que el equipo técnico en cada institución puede resolver en primera instancia (Amado-Salvatierra, Linares, García, Sánchez y Rios, 2012). Luego de haber evaluado las páginas principales de los portales web de las instituciones educativas, los resultados se pueden ver en el Cuadro 4 por cada uno de los principios de las WCAG 2.0 perceptible (P), operable (O), comprensible (C), robusto (R) y los errores HTML y CSS.

Utilizando Examinator se alcanza un promedio de 5.43 en el cumplimiento de accesibilidad de las páginas de inicio. Con la evaluación de TAW se determina que el $86 \%$ son advertencias que se deben revisar en el diseño y contenido de las páginas principales. El $11 \%$ son problemas que se deben corregir de acuerdo a los criterios de cumplimiento (éxito) de la WCAG 2.0. El $3 \%$ son puntos no verificados que requieren de un análisis manual completo para su cumplimiento. En la evaluación del contenido HTML con Markup Validation Service se tiene que el $54 \%$ son errores y el $46 \%$ advertencias. En la evaluación del contenido CSS con CSS Validation Service se tiene que el $3 \%$ son errores y el $97 \%$ advertencias.

Luego se realizó un diagrama de Pareto utilizando los valores obtenidos de la evaluación de Examinator con el fin de presentar gráficamente el porcentaje de cumplimiento de accesibilidad de las instituciones educativas de la ciudad de Cuenca, ver cuadro 4.

\begin{tabular}{|c|c|c|c|c|c|c|c|c|}
\hline \multirow{2}{*}{$\begin{array}{l}\text { Instituciones } \\
\text { educativas }\end{array}$} & \multirow{2}{*}{$\begin{array}{c}\text { Páginas } \\
\text { analizadas }\end{array}$} & \multirow{2}{*}{ Examinator } & \multicolumn{4}{|c|}{ TAW } & \multirow{2}{*}{$\begin{array}{c}\text { Markup } \\
\text { Validation } \\
\text { Service } \\
\text { Errores } \\
\end{array}$} & \multirow{2}{*}{$\begin{array}{c}\text { CSS } \\
\text { Validation } \\
\text { Service } \\
\text { Errores }\end{array}$} \\
\hline & & & $\mathbf{P}$ & $\mathbf{0}$ & $\mathbf{C}$ & $\mathbf{R}$ & & \\
\hline Aaschool & 7 & 4.6 & 26 & 12 & 3 & 75 & 10 & 4 \\
\hline adistanciabg & 7 & 8.2 & 73 & 31 & 6 & 726 & 0 & 73 \\
\hline Amauta & 7 & 4.8 & 48 & 25 & 5 & 44 & 38 & 30 \\
\hline americancollege & 7 & 4.6 & 99 & 41 & 9 & 38 & 14 & 13 \\
\hline benignomalo & 7 & 4.8 & 51 & 27 & 8 & 17 & 2 & 42 \\
\hline Casc & 7 & 5.8 & 104 & 61 & 2 & 318 & 10 & 21 \\
\hline Catalinas & 4 & 5.3 & 129 & 12 & 2 & 108 & 3 & 40 \\
\hline Comilcue & 4 & 5.6 & 74 & 24 & 6 & 11 & 288 & 201 \\
\hline
\end{tabular}

Licencia Creative Commons Atribución 4.0 Internacional (CC BY 4.0)

Revista Cátedra, 2 (2), pp. 55-75, mayo-agosto 2019. e-ISSN: 2631-2875

https://doi.org/10.29166/catedra.v2i2.1646 


\begin{tabular}{lrrrrrrrr}
\hline continental & 7 & 5.3 & 91 & 14 & 3 & 408 & 34 & 6 \\
\hline delasallecuenca & 7 & 4.6 & 192 & 16 & 2 & 159 & 30 & 51 \\
\hline herlindatoral & 7 & 5.4 & 90 & 55 & 9 & 402 & 385 & 294 \\
\hline laasuncion & 7 & 3.1 & 16 & 15 & 3 & 3 & 92 & 126 \\
\hline latinoamericano & 7 & 5.5 & 73 & 31 & 4 & 9 & 13 & 1 \\
\hline Madrid & $\underline{6}$ & 5.8 & 8 & 11 & 2 & 571 & 10 & 0 \\
\hline manueljcalle & 3 & 7.2 & 34 & 20 & 3 & 25 & 11 & 10 \\
\hline mariorizzini & 7 & 6.3 & 36 & 21 & 11 & 10 & 1 & 9 \\
\hline Santana & 7 & 5.0 & 35 & 31 & 5 & 62 & 3 & 1 \\
\hline Sscc & 7 & 4.2 & 53 & 31 & 2 & 166 & 38 & 20 \\
\hline sudamericano & 7 & 6.1 & 59 & 41 & 9 & 13 & 15 & 24 \\
\hline Uebi & 7 & 5.2 & 137 & 35 & 20 & 442 & 1 & 8 \\
\hline uelac & 7 & 5.5 & 47 & 27 & 11 & 303 & 23 & 35 \\
\hline uemo & 7 & 5.3 & 214 & 37 & 10 & 1113 & 5 & 10 \\
\hline ueoblatas & 7 & 5.9 & 41 & 30 & 5 & 170 & 21 & 9 \\
\hline uepcc & 6 & 5.7 & 27 & 20 & 13 & 452 & 5 & 19 \\
\hline ueporvenir & 7 & 5.4 & 37 & 26 & 2 & 16 & 13 & 123 \\
\hline uesmacuenca & 7 & 4.5 & 200 & 24 & 2 & 125 & 36 & 33 \\
\hline Uets & 7 & 7.5 & 72 & 31 & 7 & 661 & 28 & 12 \\
\hline unidadborja & 7 & 5.6 & 356 & 116 & 2 & 181 & 18 & 143 \\
\hline verbo & 7 & 4.7 & 38 & 25 & 5 & 71 & 39 & 18 \\
\hline ToTAL: & $\mathbf{1 9 1}$ & $\mathbf{1 5 7 . 5}$ & $\mathbf{2 4 6 0}$ & $\mathbf{8 9 0}$ & $\mathbf{1 7 1}$ & $\mathbf{6 6 9 9}$ & $\mathbf{1 1 8 6}$ & $\mathbf{1 3 7 6}$ \\
\hline Total errores / páginas: & & $\mathbf{5 . 4 3}$ & $\mathbf{1 2 . 9}$ & $\mathbf{4 . 7}$ & $\mathbf{0 . 9}$ & $\mathbf{3 5 . 1}$ & $\mathbf{6 . 2}$ & $\mathbf{7 . 2}$ \\
\hline
\end{tabular}

Cuadro 4. Análisis de accesibilidad de las páginas de inicio de las instituciones educativas de la ciudad de Cuenca

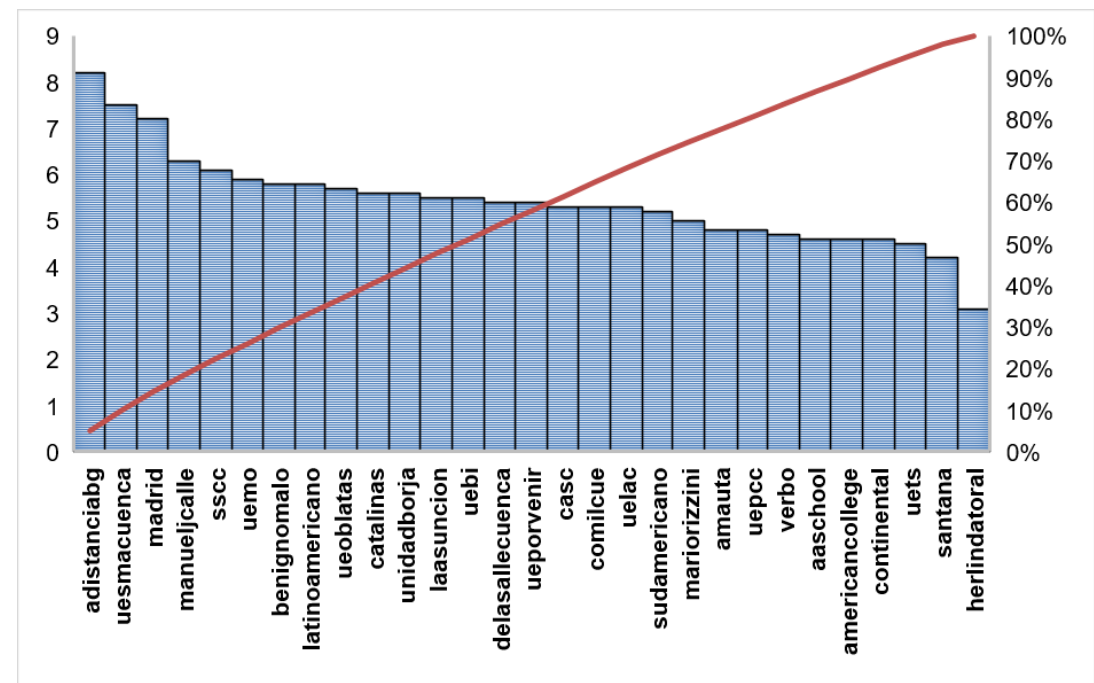

Figura 2. Diagrama de Pareto de las instituciones educativas de la ciudad de Cuenca

\subsection{Evaluación de los portales web}

Después de haber evaluado los portales web de las instituciones educativas. Se determinan los errores por cada uno de sus principios: perceptible (P), operable (O), comprensible (C) y robusto (R); y los errores HTML y CSS, como se puede ver en el Cuadro 5.

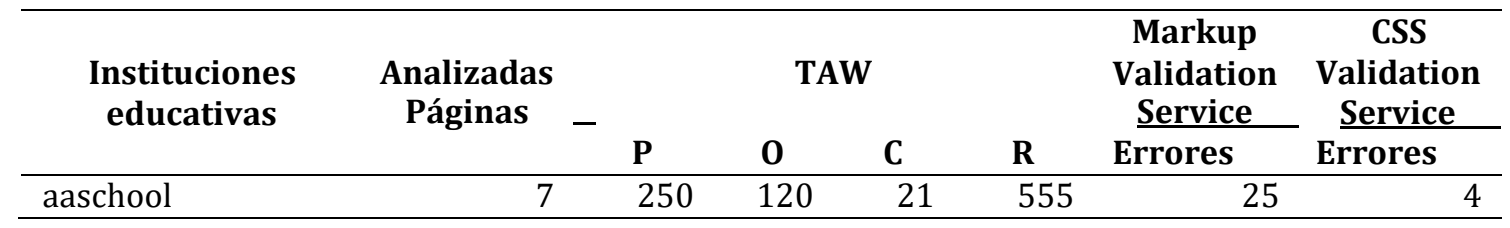




\begin{tabular}{|c|c|c|c|c|c|c|c|}
\hline adistanciabg & 7 & 332 & 191 & 49 & 3923 & 32 & 32 \\
\hline amauta & 7 & 284 & 115 & 35 & 321 & 31 & 30 \\
\hline americancollege & 7 & 320 & 248 & 64 & 367 & 6 & 25 \\
\hline benignomalo & 7 & 246 & 154 & 44 & 72 & 10 & 36 \\
\hline Casc & 7 & 342 & 189 & 30 & 2232 & 5 & 21 \\
\hline catalinas & 4 & 366 & 43 & 12 & 375 & 3 & 40 \\
\hline comilcue & 4 & 200 & 62 & 29 & 40 & 95 & 67 \\
\hline continental & 7 & 473 & 115 & 34 & 1646 & 10 & 1 \\
\hline delasallecuenca & 7 & 997 & 92 & 20 & 870 & 17 & 36 \\
\hline herlindatoral & 7 & 435 & 189 & 55 & 1949 & 313 & 237 \\
\hline laasuncion & 7 & 271 & 184 & 61 & 283 & 15 & 51 \\
\hline latinoamericano & 7 & 491 & 84 & 17 & 57 & 8 & 17 \\
\hline madrid & $\underline{6}$ & 72 & 66 & 11 & 2888 & 2 & 0 \\
\hline manueljcalle & 3 & 71 & 38 & 13 & 30 & 30 & 9 \\
\hline mariorizzini & 7 & 96 & 78 & 38 & 23 & 5 & 1 \\
\hline santana & 7 & 132 & 128 & 33 & 315 & 5 & 1 \\
\hline Sscc & 7 & 454 & 499 & 44 & 1177 & 28 & 28 \\
\hline sudamericano & 7 & 578 & 225 & 64 & 969 & 18 & 23 \\
\hline uebi & 7 & 424 & 187 & 91 & 2837 & 7 & 8 \\
\hline uelac & 7 & 390 & 202 & 76 & 1756 & 5 & 16 \\
\hline uemo & 7 & 501 & 147 & 58 & 7435 & 8 & 10 \\
\hline ueoblatas & 7 & 244 & 137 & 76 & 1026 & 13 & 6 \\
\hline uepcc & 6 & 358 & 163 & 64 & 2827 & 14 & 17 \\
\hline ueporvenir & 7 & 238 & 146 & 33 & 151 & 17 & 120 \\
\hline uesmacuenca & 7 & 704 & 126 & 52 & 552 & 29 & 34 \\
\hline uets & 7 & 736 & 252 & 43 & 3828 & 172 & 26 \\
\hline unidadborja & 7 & 712 & 466 & 38 & 1106 & 6 & 139 \\
\hline verbo & 7 & 258 & 168 & 50 & 498 & 28 & 18 \\
\hline TOTAL: & 191 & $\mathbf{1 0 9 7 5}$ & 4814 & 1255 & 40108 & 958 & 1054 \\
\hline
\end{tabular}

Cuadro 5. Análisis de accesibilidad de los portales web de las instituciones educativas de la ciudad de Cuenca

Con la evaluación de TAW se determina que el $87 \%$ son advertencias que se deben revisar en el diseño y contenido de las páginas web. El $9 \%$ son problemas que se deben corregir de acuerdo a los criterios de cumplimiento (éxito) de la WCAG 2.0. El $4 \%$ son puntos no verificados que requieren de un análisis manual completo para su cumplimiento. En la evaluación del contenido HTML con Markup Validation Service se tiene que el 52 \% son errores y el $48 \%$ advertencias. En la evaluación del contenido CSS con CSS Validation Service se tiene que el $3 \%$ son errores y el $97 \%$ advertencias.

Cabe mencionar que los errores de Markup Validation Service (HTML) y CSS Validation Service se promediaron como resultado final. Por la razón de que los errores eran muy idénticos, ya que se utilizaba el mismo código HTML y CSS en todas las páginas web del portal web. En el Cuadro 6, se puede ver el resumen de los errores de los portales web de las 29 instituciones educativas. 


\begin{tabular}{lrrrrrrr}
\hline & \multicolumn{1}{c}{ P } & \multicolumn{1}{c}{ O } & \multicolumn{1}{l}{ C } & \multicolumn{1}{c}{ R } & \multicolumn{1}{c}{ Errores HTML } & \multicolumn{2}{c}{ Errores CSS } \\
\hline Mínimo & 71 & 38 & 11 & 23 & 2 & 0 \\
\hline Mediana & 342 & 147 & 43 & 870 & 14 & 23 \\
\hline Media & 378.4 & 166 & 43.28 & 1383 & 33.07 & 36.31 \\
\hline Máximo & 997 & 499 & 91 & 7435 & 313 & 237 \\
\hline
\end{tabular}

Cuadro 6. Resumen de los errores de accesibilidad de los portales web

La mayor cantidad de errores está en el principio robusto (R) debido a que las páginas web analizadas no son lo suficientemente robustas para ser interpretados de forma fiable por una amplia variedad de agentes de usuario, incluyendo las ayudas técnicas (procesamiento, nombre, función y valor). La corrección de los errores de los lenguajes de marcado HTML o XHTML y las hojas de estilo en cascada CSS en los portales web contribuirá al cumplimiento de los principios de la WCAG 2.0 y sus niveles conformidad.

\subsection{Nivel de cumplimiento}

Luego de haber revisado los portales web de las instituciones educativas de la ciudad de Cuenca, Ecuador se verifica que ninguna tiene los iconos que certifiquen la accesibilidad de los sitios por la W3C en HTML, CSS o sus niveles de conformidad A, AA y AAA. De acuerdo con los resultados obtenidos, se determina que es necesario corregir errores en todos los portales web analizados de las instituciones educativas de la ciudad de Cuenca. Por lo tanto, es evidente su incumplimiento con la primera transitoria del reglamento técnico ecuatoriano RTE INEN 288, que establece que todos los sitios web ecuatorianos que proporcionan un servicio público deben ser accesibles WCAG 2.0 nivel A hasta el 8 de agosto de 2018. Las instituciones educativas deben emprender con responsabilidad el cumplimiento normativo de accesibilidad vigente para brindar un acceso universal a sus portales web.

\section{Conclusiones}

La mayor cantidad de errores es debido a que las páginas web analizadas no son lo suficientemente robustas. El contenido debe ser robusto para que pueda ser interpretado por una amplia variedad de agentes de usuario, incluyendo tecnologías de apoyo. Los 22 sitios web de las instituciones educativas usan sistemas gestores de $\operatorname{contenidos}^{5}$ (8 WordPress, 9 Joomla, 3 DNN y 2 Wix), los 7 restantes son diseñados y programados. La apreciación anterior da una primera impresión de la estructura y diseño de los sitios web, considerando que los resultados son preliminares; para que los resultados sean fiables y completos se deben realizar pruebas manuales con expertos.

A pesar de la importancia de la accesibilidad web, su cumplimiento en los portales web de las instituciones educativas analizadas es limitado. Se requiere corregir errores en todos los portales web de las instituciones educativas de la ciudad de Cuenca analizadas. Por el promedio de errores encontrados, es más factible que las instituciones educativas rediseñen sus portales web, a fin de cumplir con las directrices de accesibilidad web. También pueden mejorar el cumplimiento de las WCAG 2.0, con un arduo trabajo de

${ }^{5}$ Sistema gestor de contenidos (CMS): ¿Qué CMS utiliza este sitio? Obtenido de https://whatcms.org/

Licencia Creative Commons Atribución 4.0 Internacional (CC BY 4.0)

Revista Cátedra, 2 (2), pp. 55-75, mayo-agosto 2019. e-ISSN: 2631-2875

https://doi.org/10.29166/catedra.v2i2.1646 
revisión y corrección del código fuente de sus portales web. Para lo cual los desarrolladores deben comprender la accesibilidad, aprender cómo implementarla y conocer sus obligaciones legales. Los beneficios es proporcionar un acceso universal a la web y mejorar su usabilidad. Según estadísticas de la OMS (Banco Mundial, 2011, pág. 5), el CONADIS (Consejo Nacional para la Igualdad de Discapacidades, 2019) las personas con discapacidad se incrementan a pasos agigantados y la accesibilidad web es un mecanismo que permite mejorar la calidad de vida de estas personas.

La adopción de la norma NTE INEN-ISO/IEC 40500 en el Ecuador exige que los portales web de las instituciones educativas de la ciudad de Cuenca sean accesibles. Arduo trabajo que los desarrolladores de software deben emprender para cumplir con las directrices de accesibilidad web y sus niveles de conformidad. Si los desarrolladores o programadores no toman conciencia de la normativa vigente y la aplican en el desarrollo de páginas web no se estará fomentando a un acceso universal a la web. El desafío de la comunidad educativa es emprender proyectos de accesibilidad web para el cumplimiento de las WCAG 2.0 en sus sitios web con un nivel de conformidad AA como se establece en el reglamento técnico ecuatoriano RTE INEN 288 "Accesibilidad para el contenido web" hasta agosto del 2020. Una limitación de esta investigación constituye el tamaño de la muestra que se toma de los portales web de las instituciones educativas de la ciudad de Cuenca, lo que no posibilita generalizar los resultados obtenidos.

\section{Agradecimientos}

Agradecemos al Dr. Sergio Luján Mora de la Universidad de Alicante los consejos recibidos durante la elaboración de este artículo. 


\section{Bibliografía}

Acosta, T., y Luján-Mora, S. (2017). Análisis de la accesibilidad de los sitios web de las universidades ecuatorianas de excelencia. Enfoque UTE, 8(1), págs. 46-61. doi:10.29019/enfoqueute.v8n1.133

Acosta-Vargas, P., Acosta, T., y Luján-Mora, S. (2018). Challenges to Assess Accessibility in Higher Education Websites: A Comparative Study of Latin America Universities. IEEE Access, 6, págs. 36500-36508. doi:10.1109/ACCESS.2018.2848978

Acosta-Vargas, P., Luján-Mora, S., y Salvador-Ullauri, L. (2016). Evaluation of the web accessibility of higher-education websites. 15th International Conference on Information Technology Based Higher Education and Training (ITHET), (págs. 1-6). Estambul. doi:10.1109/ITHET.2016.7760703

Acosta-Vargas, P., Luján-Mora, S., y Salvador-Ullauri, L. (2017). Web accessibility polices of higher education institutions. 16th International Conference on Information Technology Based Higher Education and Training (ITHET), (págs. 1-7). Ohrid. doi:10.1109/ITHET.2017.8067808

Amado-Salvatierra, H., Linares, B., García, I., Sánchez, L., y Rios, L. (2012). Análisis de Accesibilidad Web y Diseño Web Accesible para instituciones socias del proyecto ESVI-AL. Actas del IV Congreso Internacional sobre Aplicación de Tecnologías de la Información y Comunicaciones Avanzadas, (págs. 54-61). Obtenido de http://citeseerx.ist.psu.edu/viewdoc/download?doi=10.1.1.679.9927\&rep=rep1\& type $=$ pdf\#page $=55$

Arasid, W., Abdullah, A. G., Wahyudin, D., Abdullah, C. U., Widiaty, I., Zakaria, D., . . Juhana, A. (2018). An Analysis of Website Accessibility in Higher Education in Indonesia Based on WCAG 2.0 Guidelines. \{IOP\} Conference Series: Materials Science and Engineering. 306, págs. 1-8. $\quad\{$ IOP $\}$ Publishing. doi:10.1088/1757899x/306/1/012130

Banco Mundial. (2011). Informe mundIal la dIscapacIdad. Educación, 218(219), 219. Obtenido http://www.who.int/disabilities/world_report/2011/summary_es.pdf

Campoverde-Molina, M. (2016). La accesibilidad web. Un reto en el entorno educativo ecuatoriano. Revista Científica y Tecnológica UPSE, III(3), págs. 90-98.

Campoverde-Molina, M., Vizñay-Durán, J., y Reyes-Espinosa, D. (2016). Accesibilidad web en las instituciones de salud de la ciudad de Cuenca. Análisis preliminar. IV Conferencia Internacional sobre Aplicación de Tecnologías de la Información y Comunicaciones para mejorar la Accesibilidad (ATICAcces 2016), 1, págs. 125-132. Cuenca.

Confederación de Empresarios de Andalucía. (2010a). Accesibilidad Web. Obtenido de http://webaccesible.cea.es/?q=nivelesWCAG20

Confederación de Empresarios de Andalucía. (2010b). Herramientas de evaluación automáticas. Obtenido de http://webaccesible.cea.es/?q=automaticas 
Consejo Nacional para la Igualdad de Discapacidades. (2015). Estadística personas con discapacidad. Obtenido de http://www.consejodiscapacidades.gob.ec/wpcontent/uploads/downloads/2015/09/estadistica_conadis.pdf

Consejo Nacional para la Igualdad de Discapacidades. (2019). Estadísticas de Discapacidad. Obtenido de https://www.consejodiscapacidades.gob.ec/estadisticas-dediscapacidad/

Instituto Ecuatoriano de Normalización. (2014). Tecnología de la información - Directrices de accesibilidad para el contenido web del W3C (WCAG) 2.0. Obtenido de https://drive.google.com/file/d/0Bxl4LLo2QyyJMmZDNGwzWEdHMGc/view

Instituto Ecuatoriano de Normalización. (2016). Reglamento técnico ecuatoriano RTE INEN 288 "accesibilidad para el contenido web". Obtenido de https://drive.google.com/file/d/0Bxl4LLo2QyyJdURfOHViRGpCNFU/view

International Organization for Standardization. (2012). Information technology -- W3C Web Content Accessibility Guidelines (WCAG) 2.0. Obtenido de https://www.iso.org/standard/58625.html

Luján-Mora, S. (2018a). Herramientas de revisión automática de la accesibilidad web. Obtenido de http://accesibilidadweb.dlsi.ua.es/?menu=hr-revision-automaticas

Luján-Mora, S. (2018b). Principios y directrices de WCAG 2.0. Obtenido de http://accesibilidadweb.dlsi.ua.es/?menu=principios-2.0

Ministerio de Educación del Ecuador. (2018). AMIE (Estadísticas educativas a partir de 20092010). Obtenido de https://educacion.gob.ec/amie/

Naciones Unidas. (2006). Convención sobre los derechos de las personas con discapacidad. Obtenido de http://www.un.org/disabilities/documents/convention/convoptprot-s.pdf

Network Information Center. (2019). Tipos de dominios. Obtenido de https://www.nic.ec/content.php?action=mypages\&page=TipoDominios.html

Ortiz Ruiz, Y. T. (2019). Accesibilidad en sitios web del Ministerio de Educación de Chile. Tendencias Pedagógicas, 33(2019), págs. 99-116.

Rahim Wan Mohd Isa, W. A., Hakim Suhaimi, A. I., Ariffrn, N., Fatimah Ishak, N., y Mohd Ralim, N. (2016). Accessibility evaluation using Web Content Accessibility Guidelines (WCAG) 2.0., (págs. 1-4). Melaka. doi:10.1109/IUSER.2016.7857924

Rodríguez V., L., Rosas Chávez, P., y Suárez Naranjo, C. (2019). Análisis comparativo de usabilidad en los sitios web de las universidades públicas de la ciudad de Riobamba - Ecuador. Atlante: Cuadernos de Educación y Desarrollo, febrero, págs. 1-14.

Sam-Anlas, C. A., y Stable-Rodríguez, Y. (2016). Evaluación de la accesibilidad web de los portales del Estado en Perú. Revista Española de Documentación Científica, 39(1), págs. 1-15.

Stamford Interactive. (2012). WCAG 2.0 Map. Obtenido de https://www.digitalpulse.pwc.com.au/wpcontent/uploads/2014/08/WCAG20Map.pdf

World Wide Web Consortium. (2006). Web Accessibility Evaluation Tools List. Obtenido de https://www.w3.org/WAI/ER/tools/ 
World Wide Web Consortium. (2008). Web Content Accessibility Guidelines (WCAG) 2.0. Obtenido de http://www.w3.org/TR/WCAG20/

World Wide Web Consortium. (2015). Introducción a la Accesibilidad Web. Obtenido de https://www.w3c.es/Traducciones/es/WAI/intro/accessibility

World Wide Web Consortium. (2018). Web Content Accessibility Guidelines (WCAG) 2.1. Obtenido de https://www.w3.org/TR/WCAG21/ 


\section{Autores}

MILTON CAMPOVERDE-MOLINA obtuvo su título de Magíster en Evaluación y Auditoria de Sistemas Tecnológicos en la Universidad de las Fuerzas Armadas - ESPE (Ecuador) en 2015. Obtuvo el título de Magíster en Docencia Universitaria en la Universidad de las Fuerzas Armadas - ESPE (Ecuador) en 2014. Obtuvo el título de Ingeniero de Sistemas por la Unidad Académica de Tecnologías de la Información y la Comunicación de la Universidad Católica de Cuenca (Ecuador) en 2009. Obtuvo el título de Tecnólogo Analista de Sistemas por la Unidad Académica de Tecnologías de la Información y la Comunicación de la Universidad Católica de Cuenca (Ecuador) en 2007.

Actualmente es profesor titular de la Unidad Académica de Tecnologías de la Información y la Comunicación de la Universidad Católica de Cuenca (Ecuador). Sus principales temas de investigación incluyen la accesibilidad web, discapacidades, desarrollo web, pedagogía. Es autor de capítulos de libros y artículos publicados en varias revistas.

LLORENÇ VALVERDE obtuvo la licenciatura en matemáticas (1976) por la Universidad de Barcelona y el doctorado en informática (1982) por la Universidad Politécnica de Cataluña. Catedrático de la Universidad de las Islas Baleares desde 1989, fue director del departamento de Matemáticas e informática de 1994 a 2003. De enero de 2006 a julio de 2013 fue vicerrector de Tecnología de la Universidad Abierta de Cataluña. En 1985 obtuvo el Premio Barcelona para Estudios e Investigaciones en Ciencia Cognitiva y Lógica, mientras era profesor de la Universidad Politécnica de Cataluña, de la que llegó a ser catedrático en 1988. Ha realizado diversas estancias de investigación en universidades y centros de Europa y también de los Estados Unidos. Su actividad de investigación se ha centrado en el estudio de modelos por el razonamiento aproximado y por defecto. Es autor y coautor de numerosos artículos científicos y editor de varios libros publicados por prestigiosas editoriales especializadas. Su actividad docente se ha centrado esencialmente en asignaturas relacionadas con métodos matemáticos, impartidas en los estudios de Arquitectura, Informática y Matemáticas.

Colabora habitualmente en medios de comunicación y ha publicado varios trabajos sobre las nuevas relaciones entre tecnología, cultura y educación que han establecido la digitalización y la expansión de Internet. Es autor de varios libros de divulgación científicotécnica, el último de los cuales, ha sido publicado por la Editorial UOC con el título "Siete fracasos que han cambiado el mundo: Del lavavajillas a la telefonía móvil" (2014).

Licencia Creative Commons Atribución 4.0 Internacional (CC BY 4.0)

Revista Cátedra, 2 (2), pp. 55-75, mayo-agosto 2019. e-ISSN: 2631-2875

https://doi.org/10.29166/catedra.v2i2.1646 